\title{
Treatment of pneumocystis carinii pneumonia in children
}

\author{
A. LIPSON,* W. C. MARSHALL, AND A. R. HAYWARD \\ From The Hospital for Sick Children, and Institute of Child Health, University of London
}

SUMMARY Eighteen children with pneumocystis carinii pneumonia diagnosed over a period of 16 years at a children's hospital are reviewed. All had an underlying disease, either congenital immunodeficiency or a malignancy. 15 patients were treated, 10 with pentamidine isethionate alone, 2 with both pentamidine and co-trimoxazole, and 3 with co-trimoxazole alone. 12 of the treated group recovered and the 3 untreated patients died. The 3 deaths after treatment occurred in children receiving pentamidine alone, and in whom secondary factors contributed. The side effects of treatment with pentamidine were high, and included local reactions, hypoglycaemia, and uraemia. However, our results confirm that pentamidine is an effective treatment for pneumocystis carinii pneumonia in childhood. Co-trimoxazole may be an effective and relatively nontoxic alternative treatment.

Pneumocystis carinii was first recognized as a serious lung pathogen when it was found to be the aetiological agent of epidemics of interstitial pneumonia in malnourished preterm infants from Eastern Europe in the 1940s and 1950s (Gajdusek, 1957). In Western countries it is now recognized as a rare pneumonia occurring almost exclusively in patients with immunodeficiency or malignant disease.Pneumocystis carinii is thought to be a protozoan because of its morphological appearances; it infects the terminal respiratory passages of both man and a number of animals causing diffuse alveolar disease and interstitial pneumonitis (Robbins, 1967).

\section{Patients}

Eighteen children with histologically proven pneumocystis carinii pneumonia have been diagnosed at this hospital in the 16 years from January 1959 to December 1975 (Fig. 1). Diagnosis in each case was made by examination of lung tissue by histological methods including Gomori's methenamine silver stain. All patients had an underlying disease, either congenital immunodeficiency or a malignancy.

Fifteen children were treated with either pentamidine isethionate alone, co-trimoxazole alone, or both pentamidine and co-trimoxazole. Details of these patients are given in the Table, and are grouped according to the treatment they received. 3 patients were not treated. The first was a boy aged

Received 23 August 1976

Present address: Royal Alexandra Hospital for Children, Camperdown, 2050 NSW, Australia.
6 months with variable immunodeficiency who had a post-mortem diagnosis in 1959 before treatment became available generally. The second was a boy aged 6 months with severe combined immunodeficiency who was transferred from another hospital moribund and died within 2 days, and the third was a girl aged 7 months with Letterer-Siwe disease who died unexpectedly before this diagnosis was made and specific treatment given.

Patients with malignancy. No particular treatment combination appeared to predispose these patients to the infection. Each of the 6 children with acute lymphocytic leukaemia received radiotherapy, one for a mediastinal lymphoma (Case 3), 2 for central nervous system (CNS) relapse (Cases 8, 13), and 3 (Cases 9, 10, 12) as prophylaxis. Both patients who had radiotherapy for CNS relapse had pneumonia within 11 months and 5 months of the treatment. Prednisone therapy was suspended within one month

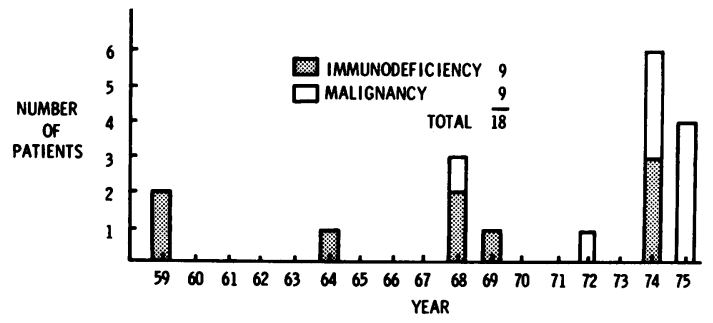

Fig. 1 Pneumocystis carinii pneumonia. The Hospital for Sick Children, Great Ormond Street, 1959-1975. 
of presentation in 4 cases, 3 within a week of diagnosis. Peripheral white cell counts at presentation of the pneumonia showed severe lymphopenia in 5 patients and neutropenia in 1 .

Patients with immunodeficiency. A family history of an affected male sib was noted in Case 11, but as 7 out of 9 of this group were boys, an X-linked disorder is probable. The episode of pneumonia in Case 2 probably represents a second infection with pneumocystis as he was first seen at the age of 7 months with a pneumonitis which responded to a combination of drugs, including pentamidine (Rodgers and Haggie, 1964). As there was no histological proof of the diagnosis, this patient's initial episode has not been included in the data.

\section{Clinical findings and investigations}

The mean duration of symptoms before presentation was $2 \cdot 5$ weeks, range 2 days to 6 weeks. When first seen all of the patients were tachypnoeic; and 10 had a cough, 6 a fever, and 4 patients with a history of less than 3 weeks had finger clubbing. All but 3 patients had clear chest to auscultation. Auscultatory signs when present were reported as scattered râles and crepitations throughout both lung fields. Arterial blood gases, taken in air at presentation in 12 patients, showed a uniform pattern of severe hypoxia and hypocapnia with little acid-base disturbance (Fig. 2). Many of the older patients, though cyanosed and tachypnoeic, had little toxicity or prostration at presentation. Chest $x$-ray changes in all were bilateral and showed similar features of a diffuse, progressively coalescing pneumonia that often spared the peripheral lung fields in the early stages.

In 14 patients diagnosis was confirmed by the histology of an open lung biopsy. Pneumothorax occurred after lung biopsy in one child. It was nontension in type, occurring 2 days after the procedure, and was successfully treated by underwater drain. A second child required postoperative ventilation as a result of oversedation from anaesthetic premedication. 4 patients were diagnosed by post-mortem histology of lung tissue.

\section{Deaths in treated patients}

Three patients died, 2 during and one 2 weeks after a course of pentamidine isethionate. The first (Case 3) was a child with leukaemia who had initial cytoxic drug treatment for a presumed malignant infiltration of the lungs, and died 2 days after pentamidine treatment was started. The second (Case 5), who also had leukaemia, had the lowest $\mathrm{Po}_{2}$ recorded at presentation $(2.92 \mathrm{kPa}(22 \mathrm{mmHg})$ in air) and who died of hypoxia and renal failure on the 10th day of treatment. Histology of the lung at necropsy showed unresolved pneumocystis pneumonia and an adenovirus was grown from postmortem lung. The third (Case 8) was a child with severe combined immunodeficiency who died 2 weeks after a 14-day course of pentamidine was completed. During this period he was malnourished with a serum albumin of $8 \mathrm{~g} / \mathrm{l}$. Post-mortem lung histology showed a bronchopneumonia superimposed on a resolving pneumocystis pneumonia.

Seven of the 12 patients who recovered from the pneumonia have since died of their underlying disease. Of the 5 survivors, 4 have acute lymphocytic leukaemia in remission and one has variable immunodeficiency. This latter patient is now aged 17 and was the first child reported with immunodeficiency successfully treated with pentamidine (Marshall et al., 1964).

\section{Discussion}

Our data confirm that pentamidine isethionate is an effective drug for the treatment of pneumonia caused by Pneumocystis carinii, and compares

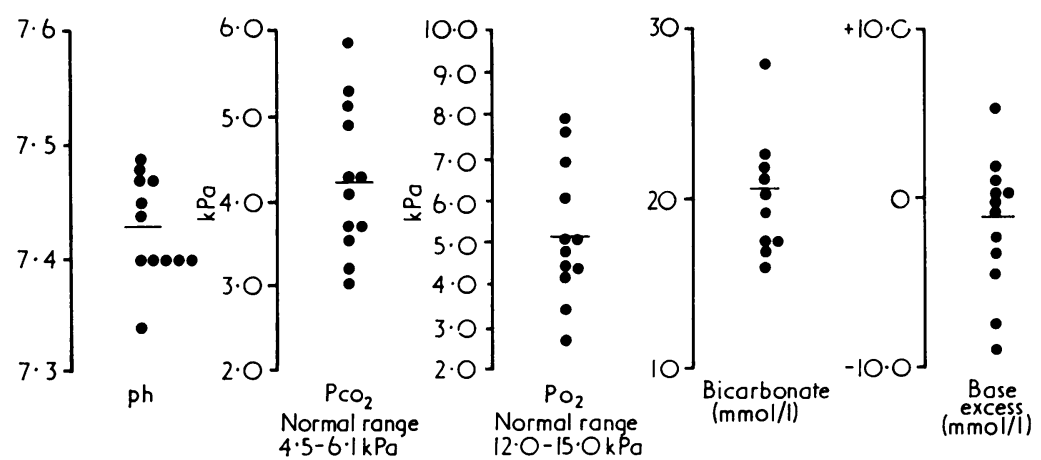

Fig. 2 Blood gases (in air) at presentation in 12 patients. Conversion-SI to traditional units: $1 \mathrm{kPa} \approx 7.5 \mathrm{mmHg}$. Bicarbonate and base excess: $1 \mathrm{mmol} / \mathrm{l} \approx 1 \mathrm{mEq} / \mathrm{l}$. 
Treatment of pneumocystis carinii pneumonia in children 317
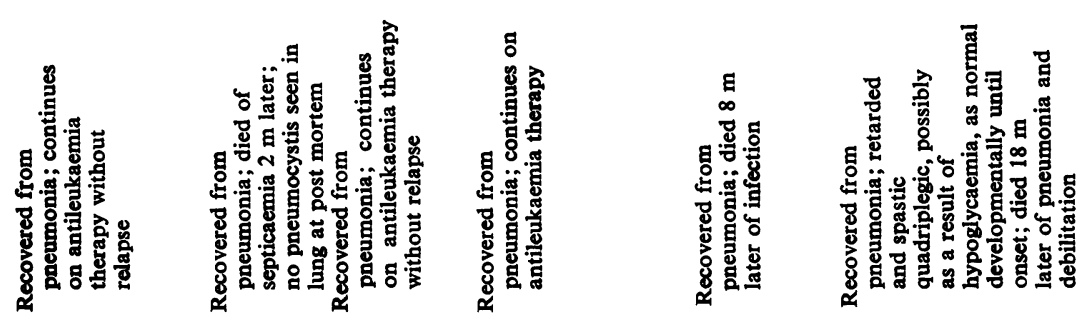

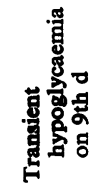

$\bar{z} \quad \bar{z} \quad \bar{z}$
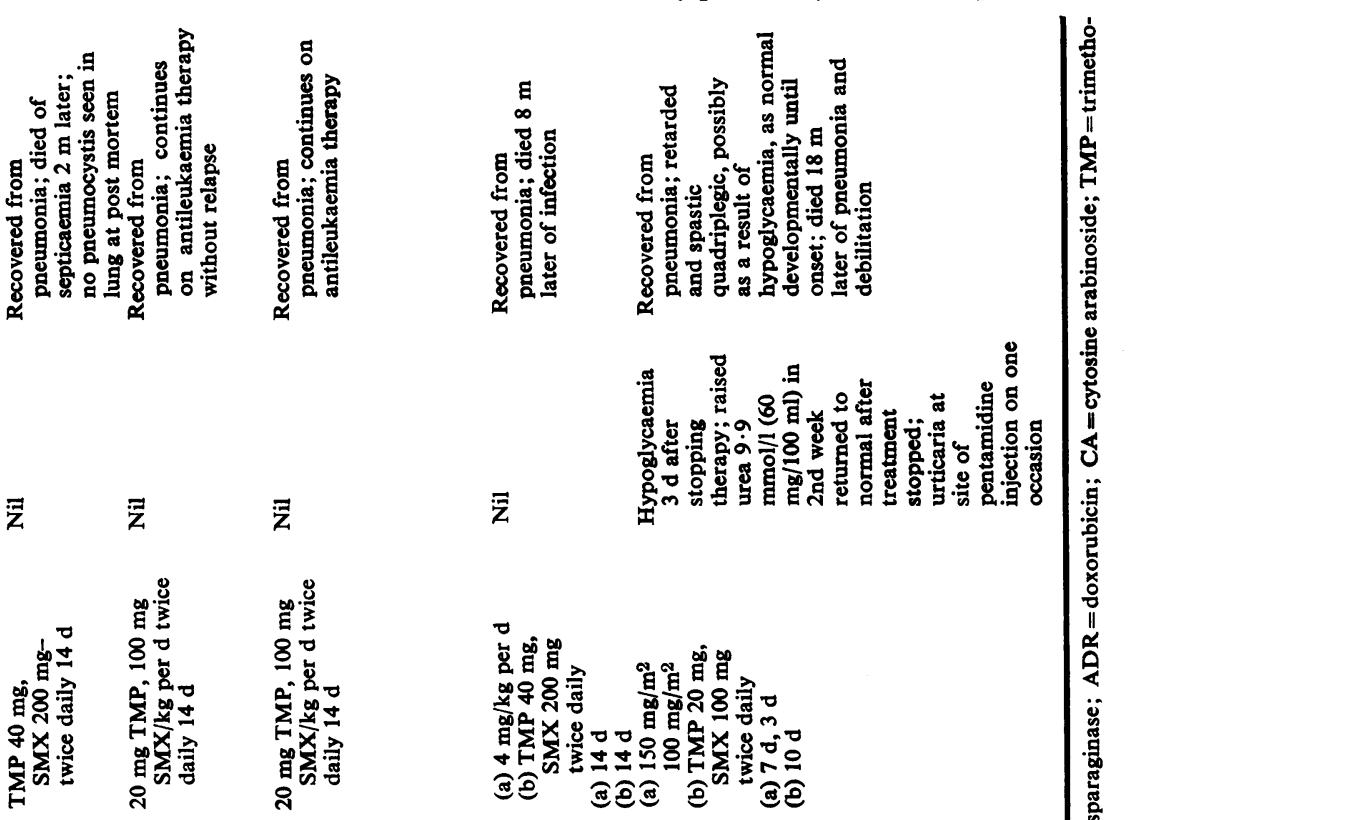

-

$\begin{array}{lll}\Xi & \Xi & 5 \\ \stackrel{5}{9} & \stackrel{4}{9}\end{array}$

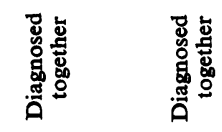

象
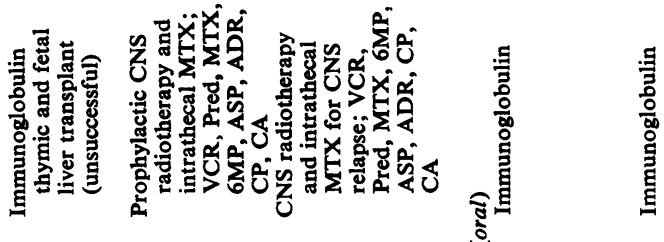

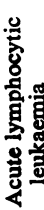
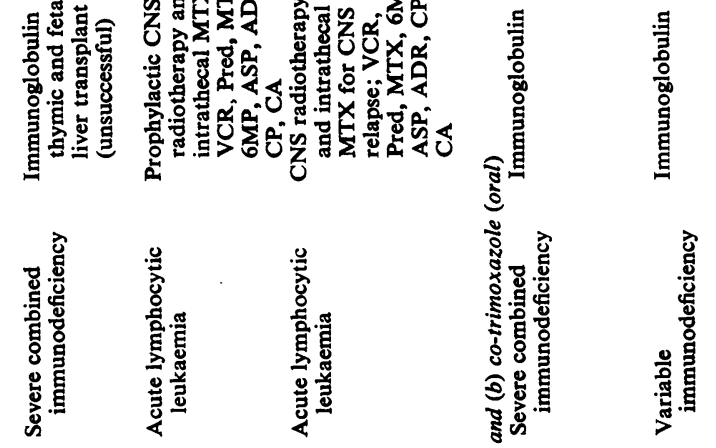

$\frac{5}{5}$

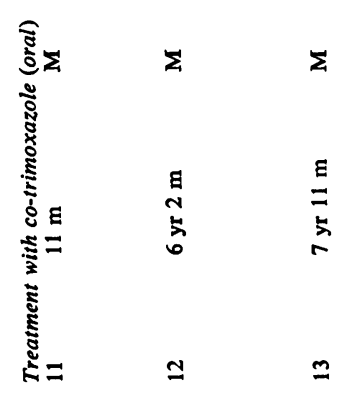

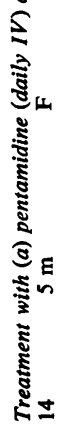

$\Sigma$

9

$\underline{m}$

$\cong$ 
favourably with the response to this drug seen in other series (Johnson and Johnson, 1970; Geelhoed et al., 1973; Hughes et al., 1973; Walzer et al., 1974; Western et al., 1975). Secondary factors in the 3 children who died reduced the possibility of a favourable response to this drug. One was given initial cytotoxic therapy in error, the disease progressing to a fulminating picture from which the patient died after 2 days' treatment (Case 3). A second child who died on the 9th day of therapy had the most severe initial picture as judged by blood gas analysis, and had recurrent episodes of hypotension and hypoxia in addition to renal tubular necrosis (Case 8). A superimposed adenovirus infection might have further compromised this patient. The third child died after a course of therapy was complete (Case 5). He was malnourished with the extremely low terminal serum albumin of $8 \mathrm{~g} / \mathrm{l}$. Severe malnutrition in itself can predispose to pneumocystis pneumonia (Hughes et al., 1974a) and might have perpetuated it in this child. There was also a bilateral bronchopneumonia which would have contributed to his demise. These 3 patients received pentamidine $4 \mathrm{mg} / \mathrm{kg}$ per day, which is a slightly lower dosage regimen than that recently suggested of $150 \mathrm{mg} / \mathrm{m}^{2}$ for 3 days followed by $100 \mathrm{mg} / \mathrm{m}^{2}$ for 7 days (Hughes et al., 1973), particularly in the younger age groups.

Pentamidine has significant side effects and those noted in this series are comparable with other reports (Johnson and Johnson, 1970; Wang et al., 1970; Geelhoed et al., 1973; Hughes et al., 1973; Walzer et al., 1974; Western et al., 1975). In experimental animals pentamidine is quickly cleared from the blood and preferentially stored in the kidney and liver. It is slowly excreted in the urine and bile, traces still being detected up to 8 months later (Waalkes et al., 1970). It is a diamidine, one of a group of drugs first developed as hypoglycaemic agents and used in tropical protozoal infections such as trypanosomiasis (Schoenbach and Greenspan, 1948). Ivády et al. (1967) first reported its efficacy in pneumocystis pneumonia. The extremely slow excretion could have accounted for a late onset of hypoglycaemia seen in one patient (Case 15) in whom it occurred 3 days after treatment was stopped. Renal tubular necrosis has been associated with the drug (Wang et al., 1970; Hughes et al., 1973; Western et al., 1975), but we are reluctant to attribute the tubular necrosis seen in Case 8 solely to renal toxicity of pentamidine, because of the associated severe hypoxia and hypotension and the fact that regeneration was seen at necropsy despite continuing therapy. A transient rise in blood urea was seen in 2 patients (Cases 6, 15). Hypotension is reported as a complication of pentamidine therapy, usually acutely after intravenous therapy. The neutropenia and thrombocytopenia seen after a prolonged treatment regimen of 35 days in one patient (Case 4) was probably secondary to a disturbance in folate metabolism which has been described with the use of pentamidine (Robbins, 1967).

Co-trimoxazole was proposed as a therapeutic agent in pneumocystis pneumonia when it was shown to be effective in animal studies (Hughes et al., 1974b), and when early clinical studies with the related combination of pyrimethamine and sulphadiazine showed promise (Kirby et al., 1971). A recent report confirms its efficacy, particularly in a high-dose regimen early in the course of infection (Hughes et al., 1975). Co-trimoxazole has many attractions as a preferred form of therapy in pneumocystis carinii pneumonia. It seems to have a rapid onset of action, a response often occurring in the first few days; and a low incidence of toxic side effects when compared with pentamidine. However, because of pentamidine's proven efficacy in this otherwise fatal infection, comparisons with cotrimoxazole are necessary before it can be discarded. The 3 patients treated with co-trimoxazole alone all recovered without side effects. 2 were treated by a high dose and one by a standard dose regimen. Co-trimoxazole might also be useful in prophylaxis, such as in the treatment of at risk contacts of a known case.

No specific cytotoxic drug combination was associated with the onset of disease. Radiotherapy was used in all the leukaemic patients. The recorded association of the onset of infection after the use of prednisone is confirmed (Rifkind et al., 1966; Johnson and Johnson, 1970; Western et al., 1975). The reason for this is not certain but might be due to a temporary suppression of the inflammatory response of the alveoli to the organism, a pre-existing infection becoming manifest after stopping this drug.

The frequent insidious onset of the symptoms of lung disease, the relative lack of toxicity, and an absence of auscultatory signs in the chest often resulted in late presentation of these children. 4 of the children had finger clubbing after cough and dyspnoea of less than 2 weeks' duration. This possibly indicates significant lung involvement before symptoms became evident.

There was no evidence, such as simultaneous clinic visits or common ward residence, to suggest cross infection in these cases. However, as man to man transmission has been strongly implied in some reports (Ruskin and Remington, 1967; Brazinsky and Phillips, 1969; Singer et al., 1975) patients with suspected infection should be isolated from other susceptible children. 
The increased numbers of patients in whom this infection has been diagnosed in recent years (Fig. 1) could be due to a greater awareness of the condition in addition to the more aggressive treatment regimens of children with malignancy by both chemotherapy and radiotherapy, resulting in a greater and sustained immunosuppression. The possibility, however, cannot be excluded that it represents an increased prevalence of the infection. Little is known of the epidemiology of Pneumocystis carinii though it is of interest to note that the epidemic form of the infection seen in Eastern Europe two and three decades ago has declined in recent years.

We are very grateful to Dr. Judith Chessells for permission to report cases under her care; and thank Dr. E. N. Hey, Dr. P. Phelan, and Professor J. F. Soothill for suggestions; Dr. A. Risdon for review of histological sections; and Miss C. Vincent for secretarial assistance.

\section{References}

Brazinsky, J. H., and Phillips, J. E. (1969). Pneumocystis pneumonia. Transmission between patients with lymphoma. Journal of the American Medical Association, 209, 1527.

Gajdusek, D. C. (1957). Pneumocystis carinii etiologic agent of interstitial plasma cell pneumonia of premature and young infants. Pediatrics, 19, 543-565.

Geelhoed, G. W., Powell, J. L., Doppman, J. L., and DeVita, V. T. (1973). Pneumocystis carinii pneumonia in cancer patients following immunosuppressive chemotherapyresults of pentamidine treatment. American Review of Respiratory Diseases, 107, 1092.

Hughes, W. T., Price, R. A., Ho-Kyun, K., Coburn, T. P., Grigsby, D., and Feldman, S. (1973). Pneumocystis carinii pneumonitis in children with malignancies. Journal of Pediatrics, 82, 404-415.

Hughes, W. T., Price, R. A., Sisko, F., Havron, W. S., Kafatos, A. G., Schonland B., and Smythe, P. M. (1974a). Protein-calorie malnutrition. A host determinant for pneumocystis carinii infection. American Journal of Diseases of Children, 128, 44-52.

Hughes, W. T., McNabb, P. C., Makres, T. D., and Feldman, S. (1974b). Efficacy of trimethoprim and sulfamethoxazole in the prevention and treatment of pneumocystis carinii pneumonitis. Antimicrobial Agents and Chemotherapy, 5, 289-293.

Hughes, W. T., Feldman, S., and Sanyal, S. K. (1975). Treatment of pneumocystis carinii pneumonitis with trimethoprim-sulphamethoxazole. Canadian Medical Association Journal, 112, (special no.), 47-50.

Ivády, G., Páldy, L., Koltay, M., Tóth, G., and Kovács, Z. (1967). Pneumocystis carinii pneumonia. Lancet, 1, 616-617.

Johnson, H. D., and Johnson, W. W. (1970). Pneumocystis carinii pneumonia in children with cancer. Journal of the American Medical Association, 214, 1067-1073.

Kirby, H. B., Kenamore, B., and Guckian, J. C. (1971). Pneumocystis carinii pneumonia treated with sulphadiazine and pyrimethamine. Annals of Internal Medicine, 75, 505-509.
Marshall, W. C., Weston, H. J., and Bodian, M. (1964). Pneumocystis carinii pneumonia and congenital hypogammaglobulinaemia. Archives of Disease in Childhood, 39, 18-25.

Remington, J. S., and Anderson, S. E. (1973). Diagnosis and treatment of pneumocystosis and toxoplasmosis in the immunosuppressed host. Transplantation Proceedings, 5, $1263-1270$.

Rifkind, D., Faris, T. D., and Hill, R. B. (1966). Pneumocystis carinii pneumonia. Annals of Internal Medicine, 65, 943-956.

Robbins, J. B. (1967). Pneumocystis carinii pneumonitis-a review. Pediatric Research, 1, 131-158.

Rodgers, T., and Haggie, M. H. N. (1964). Pneumocystis carinii pneumonia associated with hypogammaglobulinaemia responding to pentamidine. Lancet, 1, 1042.

Ruskin, J., and Remington, J. G. (1967). The compromised host and infection pneumocystis carinii pneumonia. Journal of the American Medical Association, 202, 10701074.

Schoenbach, E. B., and Greenspan, E. M. (1948). The pharmacology, mode of action and therapeutic potentialities of stilbamidine, pentamidine, propamidine and other aromatic diamidines. Medicine (Baltimore), 27, 327-377.

Singer, C., Armstrong, D., Rosen, P. P., and Schottenfeld, D. (1975). Pneumocystis carinii pneumonia-a cluster of 11 cases. Annals of Internal Medicine, 82, 772-777.

Waalkes, T. P., Denham, C., and De Vita, V. T. (1970). Pentamidine: clinical correlations in man and mice. Clinical Pharmacology and Therapeutics, 11, 505-512.

Walzer, P. D., Perl, D. P., Krogsted, D. J., Rawson, P. G., and Schultz, M. G. (1974). Pneumocystis carinii pneumonia in the United States. Annals of Internal Medicine, 80, 83-93.

Wang, J. J., Freeman, A. I., Gaeta, J. F., and Sinks, L. F. (1970). Unusual complications of pentamidine in the treatment of pneumocystis carinii pneumonia. Journal of Pediatrics, 77, 311-314.

Western, K. A., Perera, D. R., and Schultz, M. G. (1975). Pentamidine isethionate in the treatment of pneumocystis carinii pneumonia. Annals of Internal Medicine, 73, 695-702.

Correspondence to Dr. W. C. Marshall, Institute of Child Health, Guilford Street, London WC1.

\section{Addendum}

Since the preparation of this paper 4 additional cases of pneumocystis carinii pneumonia, confirmed by lung biopsy, were seen between March and August 1976. 3 were children with acute lymphocytic leukaemia who were receiving maintenance chemotherapy for the disease and had been in remission between 9 and 15 months: their total lymphocyte counts were low, 3 ranging between 400 and $700 / \mathrm{mm}^{3}\left(0.4-0.7 \times 10^{9} / \mathrm{l}\right)$. The fourth patient, a male aged 6 months, had an immunodeficiency disorder (low IgG and IgA, normal IgM, normal tests for cell-mediated immunity) and presented with respiratory symptoms, the immunodeficiency and lung infection being diagnosed during the same admission. All 4 children have recovered after treatment with co-trimoxazole $(120 \mathrm{mg} / \mathrm{kg}$ per d) administered orally twice daily for 14 days. The infant with immunodeficiency also received immunoglobulin replacement therapy. 